\title{
The effects on gait speed and activity level of a novel body weight supported treadmill training program on a 39-year-old patient with right hemiparesis
}

\begin{abstract}
Background and Purpose: Stroke is a disease that can cause neurological impairments that can affect a patient's ability to ambulate. Research suggests that body weight supported treadmill training (BWSTT) may improve gait speed and reduce the risk of falls for those with difficulty ambulating. The purpose of this case report is to describe the effects of a novel BWSTT device on the gait speed and fall risk on a 39-year-old patient who had suffered a stroke.

Case description: The patient was a 39-year-old female who had suffered a stroke resulting in right side hemiplegia. Over a 4-week period, the patient completed seven sessions of BWSTT with the GlideTrak. The duration of each session and the intervals within the session were performed at the patient's tolerance for treadmill speed and duration of the interval.

Outcomes: Improvement of $35 \%$ on the Activities-specific Balance Confidence (ABC) Scale and $25 \%$ on the Timed Up and Go (TUG) indicate that the intervention may have lowered the risk for falls but a $14 \%$ increase in the 10 Meter Walk Test time indicated that the intervention didn't improve the patient's gait speed as predicted.

Discussion: This case report indicates that one month of body weight supported treadmill training using the GlideTrak does not improve gait speed in a patient with hemiplegia, but is shown to have a positive effect on patient's balance. This intervention may have a role in maintaining a patient's cardiovascular and overall fitness, but should not be the sole intervention to improve gait speed.
\end{abstract}

Volume 3 Issue 3 - 2018

\author{
Andrew Concor PT, DPT, MBA, CIE, Angela \\ Stagliano PT, DPT, OCS, CSCS \\ Certified Industrial Ergonomist/ Physical Therapist, University of \\ South Florida, USA
}

\begin{abstract}
Correspondence: Andrew Concors, Certified Industrial Ergonomist/ Physical Therapist, University of Montana, USA, Tel: (760) 8I5-7I03, Email ergo@cptconsulting.net
\end{abstract}

Received: June 06, 2018| Published: June 12, 2018

\section{Introduction}

According to the American Stroke Association, approximately 800,000 Americans suffer a stroke annually. ${ }^{1}$ In the United States, strokes cause between $2-3 \%$ of disabilities in adults. 1 Cheung defines stroke as a non-traumatic, focal, vascular-induced injury of the central nervous system (CNS) and typically results in permanent damage in the form of cerebral infarction,

intracerebral hemorrhage ( $\mathrm{ICH}$ ) and/or subarachnoid hemorrhage $(\mathrm{SAH}) .^{2}$ Another name for stroke is cerebral vascular accident (CVA) and an individual who has a stroke can suffer a variety of motor, sensory, perceptual and behavioral impairments. Depending on the area of the brain affected these impairments can lead to a lifetime of disability as well as increase risk for further injury such as falls. ${ }^{3}$ One of the greatest disabilities associated with a CVA is altered gait speed, which has been shown to be inversely proportional to fall risk and gait impairments. ${ }^{4}$

In a systematic review, Veerbeek et al., ${ }^{5}$ characterized physical therapy interventions for stroke into five main classifications: gait and mobility interventions; interventions to improve arm and hand function; activities of daily living interventions; fitness related interventions; and interventions that don't match any of the above. ${ }^{5}$ Of the many possible gait and mobility interventions, Body Weight Supported Treadmill Training (BWSTT) is a relatively new intervention. It has been used on patients with a variety of neurological conditions such as

Cerebral Palsy, Parkinson's disease, and Spinal Cord Injury in addition to stroke. ${ }^{6-8}$ The intervention involves supporting the trunk and upper body via an inflated bladder, harness or saddle-like support that allows the patient to be suspended and aid in ambulation. The amount of support varies depending on the needs of the user or the goals of treatment. Different goals include: body weight support to compensate for pain or lower extremity weakness, to provide support for individuals with balance impairments or fear of falling, improving gait velocity, and endurance for those with neurological conditions resulting in motor control impairments.

There is currently no strong evidence for the efficacy of BWSTT in rehabilitation. A recent Cochrane review of BWSTT showed short term increases in gait velocity and endurance, but no carry over effect. ${ }^{9}$ BWSTT has been shown to have superior outcomes regarding gait speed compared to cycle ergometry with or without concomitant strength training in stroke patients. ${ }^{10}$ Charalambos et al., ${ }^{11}$ concluded that there was no significant difference between

BWSTT and other interventions. ${ }^{11}$ Veerbeek et al showed significant effect size for use of body weight support treadmill training for improving gait speed.There are several rationales for the use of BWSTT for improving gait function in stroke patients. One rationale is the concept of mass practice, where multiple repetitions of a movement drive changes in corticomotor stimulation. ${ }^{12}$ Another rationale invokes the concept of a central pattern generator (CPG) that creates reflex like movement of the limbs that can be used to restore ambulation capabilities in those that have lost voluntary control of the limbs. ${ }^{13}$ 
Each tester can alter the parameters used that may influence the effectiveness of the intervention including amount of body weight support, speed and duration of the treadmill training, frequency of training sessions as the overall length of the episode of care. In a metaanalysis, Veneri et al., ${ }^{14}$ noted that several studies used a protocol of 20 to 25 -minute sessions 4 to 5 times a week for 4 to 6 weeks had the greatest effect on gait speed.14 Chan and Patten's focused review of the literature noted body weight support (BWS) in the range of $10 \%$ to $40 \%$ at the beginning of the intervention. ${ }^{15}$ Some protocols reduced BWS as the subjects tolerated increased weight bearing. In a subsequent pilot study, they showed that increased treadmill velocity reduced gait abnormalities at slower gait speeds.

Despite the evidence that physical rehabilitation has been shown to improve the functional abilities of those suffering impairments due of a stroke, currently there is no consensus on the best approach to achieve the optimal outcomes and quality of life for patients. There is some support for the use of BWSTT to improve patients' ability to ambulate and reduce the risk of comorbidities. The purpose of this case report is to describe the effects of a novel BWSTT system on the gait velocity in a 39-year-old patient with right hemiplegia caused by a stroke.

\section{Case description}

At the time of the intervention the patient was a 39-year-old Hispanic female who suffered a series of strokes in beginning in March 2014 that resulted in speech impairments as well as right sided hemiplegia. She initially received inpatient rehabilitation for several months followed by home health physical therapy. She was then referred to outpatient rehabilitation to further improve her gait and mobility until she was discharged due to lack of progress. After her dismissal from physical therapy services she sought continued rehabilitation in Tijuana, BC, Mexico. One year ago, she was referred by her primary care physician for outpatient physical therapy at Family Health Centers of San Diego to improve her gait and general mobility. She has since received approximately 33 individualized outpatient treatment sessions, focusing on functional mobility training, gait training, balance activities, and strength and motor control training.

Currently, the patient lives with her 11-year-old daughter, brother and sister in a single story, first floor apartment. She ambulates for short periods without assistive devices, but otherwise uses a single point cane for longer distances. Approximately one week prior to the beginning of the intervention she was issued a Walkaide functional electrical stimulation neuroprosthesis (Innovative Neurotronics, Reno, NV, USA) for assisting right ankle dorsiflexion during ambulation. She is independent in the activities of daily living (ADLs) of eating, bathing, dressing, toileting, transferring and continence as well as instrumental activities of daily living (IADLs) such as using a telephone, doing laundry, and handling her own finances. The patient is not working currently due to her disability, doesn't smoke, drink alcohol or use nonprescribed drugs.

The patient's medical history includes atrial septal defect, which was repaired in 2014 as well as the above noted history of stroke. Her current medications include: Baclofen, aspirin, cholesterol medication, and antihistamine for allergies. The patient appears to be a good candidate for the body weight supported treadmill training with the goal of improving the patient's gait speed to reduce her fall risk and allow her to be more of a community ambulater.

\section{Examination and evaluation}

During the initial evaluation the patient reported no pain or other discomfort but noted difficulty getting the neuroprosthetic ankle foot orthosis (AFO) functioning properly. Gait abnormalities noted included foot flat initial contact on the right (Figure 1) forward trunk bending at mid-stance (Figure 2) hip and knee flexion during preswing (Figure 3) and lack of trunk rotation throughout the gait cycle (Figure 4). In Table 1 the initial outcomes are listed for the Activitiesspecific Balance Confidence (ABC) Scale, the Timed Up and Go Test (TUG) and 10-meter walk test (10MWT). ${ }^{16}$

Table I Outcome measures initial scores

\begin{tabular}{ll}
\hline Scale & Score \\
\hline ABC & 32 \\
TUG & 34.36 seconds \\
IOMWT & .35 meters per second \\
\hline
\end{tabular}

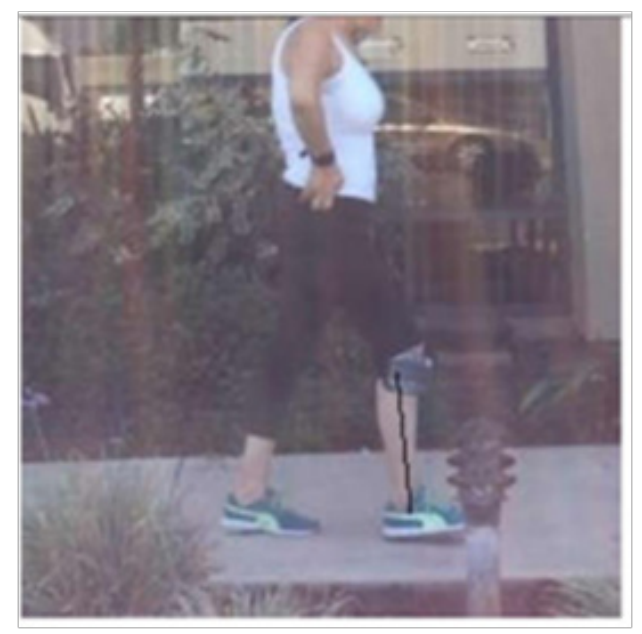

Figure I Initial contact.

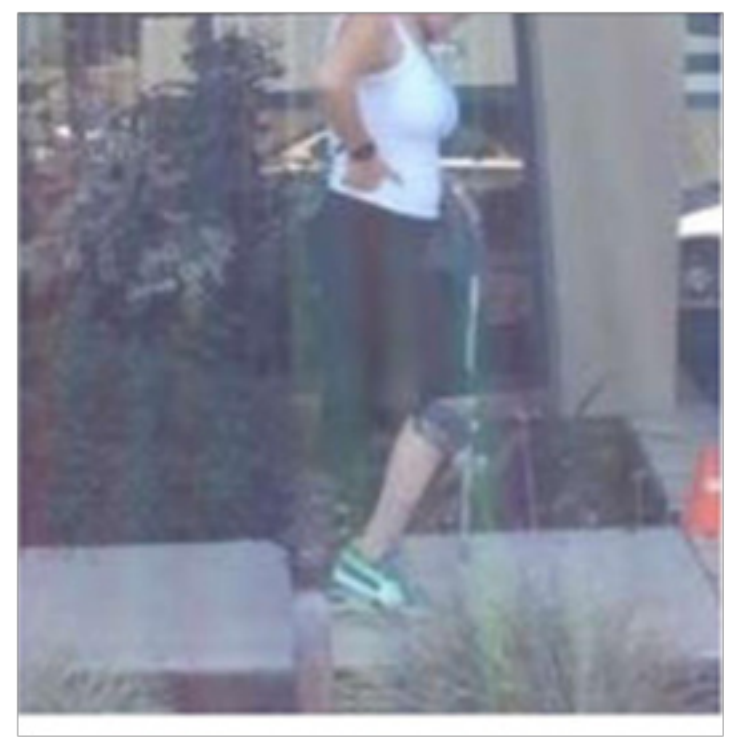

Figure 2 Pre-swing. 


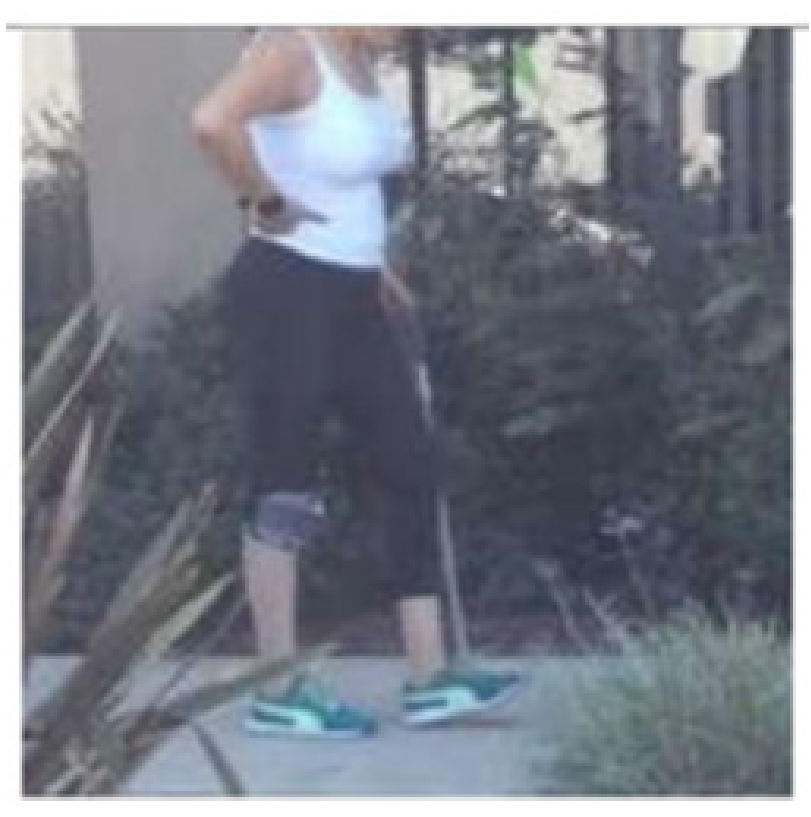

Figure 3 Midstance.

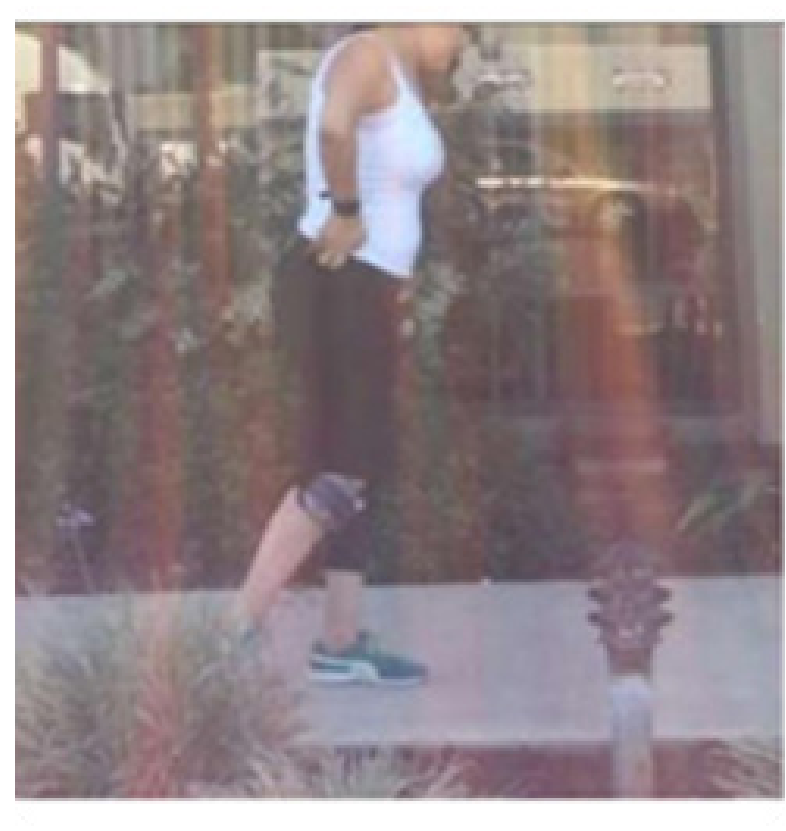

Figure 4 Terminal stance.

\section{Test and Measures}

\section{Activities-specific balance confidence scale}

The Activities-Specific Balance Confidence Scale is a questionnaire that asks the subject to rate their confidence in their abilities to maintain balance and avoid falling in 16 different scenarios. Confidence is measured by having the subject estimate their confidence in a decile percentage from $0 \%$ to $100 \%$, where $0 \%$ indicates a complete lack of confidence and $100 \%$ indicates complete confidence. Botner et al., ${ }^{17}$ reported a test-retest reliability of .85 ICC (95\% CI $0.68-0.93)$ after 4 weeks in a population of stroke patients. ${ }^{17}$ The standard error of the mean (SEM) was 6.81 and no there is no information on minimal detectable change or minimal clinically important difference. ${ }^{17}$

\section{Timed up and go}

The timed up and go (TUG) is a measure of an individual's ability to assess general mobility, ambulation, balance and fall risk in varies patient populations. The test consists of the subject sitting in a chair and, upon command, stands, walks 3 meters, turns around and returns to the chair, using the patient's typical assistive device if needed. The timing starts with the command and terminates once the subject's buttocks touches the chair. The score is the average time taken from 3 trials. Hafsteinsdóttir et al reported excellent intrarater and interrater reliability (intraclass correlation coefficient $[\mathrm{ICC}]>0.95$ ) in a systematic review of the use of the TUG with stroke patients. ${ }^{18}$ Flansbjer et al reported a Smallest Real Difference (SRD) of 23\% in a similar population.

\section{Timed I 0-meter walk test}

Timed 10-Meter Walk Test is a measurement tool for estimating gait speed that has been applied to several patient populations and is a crucial factor in determining fall risk. The test consists of placing markers at 0 meters, 2 meters, 8 meters and 10 meters. The patient is instructed to start walking at their fastest comfortable pace from the first to the last cone. The time taken from the 2 meters to 8 meters cones is measured for a total of 6 meters as the first and last two meters are not used to account for acceleration and deceleration. A series of 3 trials are completed and the average is taken. Gait velocity is computed by dividing the average time by 6 . A further conversion to miles per hour (MPH) can be made by multiplying the result by 2.23694. Perera et al reported a Standard Error of Measurement (SEM) of $0.04 \mathrm{~m} / \mathrm{s}$ and a small meaningful change of $0.06 \mathrm{~m} / \mathrm{s}$ and a substantial meaningful change $=0.14 \mathrm{~m} / \mathrm{s} .{ }^{19}$ Flansbjer et al. ${ }^{20}$ reported Intraclass Correlation Coefficient (ICC) of 0.97 when assessing fast gait speeds in stroke patients although the measured distance was 10 meters versus 6 meters as used in this paper. ${ }^{20}$

\section{Intervention}

The physical therapy intervention selected for this patient was the GlideTrak body weight support system (Figure 5). The GlideTrak uses a bicycle like seat support under the user's posterior hips on the ischial tuberosities and a counter pressure bar resting anteriorly close to or on top of the user's anterior superior iliac spines. ${ }^{16}$ The support system is then suspended from webbed belts attached to a superstructure made of aluminum tubing. ${ }^{16}$ This superstructure is placed over the treadmill to provide varying amounts of support to the user, resulting in reduction in weight bearing on the lower extremities. There is an optional shoulder harness that can be attached to the superstructure above the user for those who lack trunk control. Figures 6, Figure 7 depict the patient in the device from the side and from behind respectively.

After competition of the evaluation, it was determined that the patient have 8 sessions of BWSTT over a 4 -week period on the GlideTrak. This was in addition to her ongoing weekly outpatient physical therapy appointments with her primary physical therapist. The goals of the outpatient sessions were to improve the patient's right upper and lower extremity strength, dynamic balance, functional mobility related to sit to stand transfers and improving her gait pattern. Sessions were held at the GlideTrak training facility as device was not available at the physical therapy clinic nor were there personnel 
trained in its use. The sessions were supervised by the author, but initial set up and adjustments were performed by Glide Trak personnel until the author was confident in device set-up. Although the sessions were supervised by a licensed physical therapist they were not considered part of the patient's regular episode of care and there was no charge to the patient. There were no other financial agreements between the patient, author or the company.

Each session lasted from 45 minutes to an hour consisting of setting up the harness support and adjusting the treadmill angle to optimize weight bearing while minimizing gait abnormalities. The body weight support couldn't be calculated directly, so adjustments were made based on the patient's report of weight bearing and the evaluator's observation of gait abnormalities. The treadmill angle was between $3 \%$ and $5 \%$ for each session and adjusted to fine tune the amount of body weight support.

After initial adjustment the patient completed three to five intervals of BWSTT. The number of intervals and the duration of each interval depended on the patient's report of fatigue and changes in her gait pattern. Compensatory motions noted when the patient was fatigued were: failure to clear the toes during swing phase; trunk sway or an increase in hip adduction at mid stance. Ratings of perceived exertion (RPE) were not used, but the patient did not exhibit signs of shortness of breath and was able to carry on a conversation during the intervals. Her heart rate never exceeded 100 BPM at the end of the intervals



Figure 5 GlideTrak setup.



Figure 7 Patient setup in GlideTrak rear view. during measurement via an optical heart rate monitor and was unlikely affected by her medications.

The speed of the treadmill was adjusted for each session and interval based on the patient's tolerance as well as the above noted gait abnormalities. The patient was cautioned that the treadmill speed should not cause gait deviations or undue fatigue. The sessions and intervals were non-structured due to several reasons: the lack of consensus on an optimal protocol in the literature, the novel use of the device on a stroke patient as well as the patient's desire to get maximum benefit from the intervention. In order to calculate a quantifiable training load the maximum speed achieved in each interval was multiplied by the time of each interval. The interval loads were summed for each session to track training load over the time of the intervention. Figure 8 shows a chart of each session with the intervals for that session depicted with the width of the column displaying the maximum speed and the height depicting the duration of the interval and a trend line of the training load over the time of the intervention.

Figure 9 displays the maximum treadmill speed achieved per session with a trend line superimposed. Although the original plan for the interventions was for 8 sessions of BWSTT, the patient missed one visit due to a family emergency. Also, the last session occurred immediately after the final assessment and therefore was not included in the outcome measures.

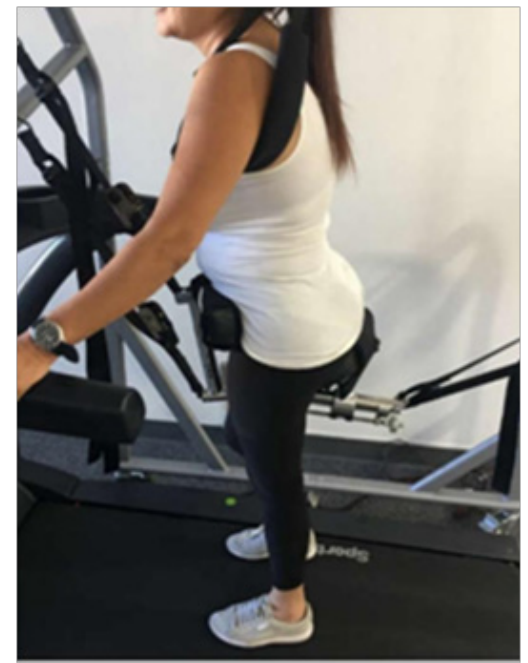

Figure 6 Patient setup in GlideTrak side view.

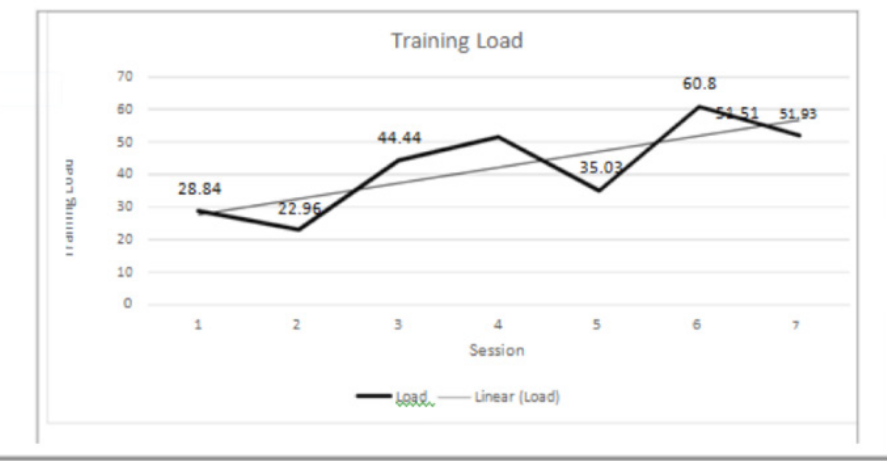

Figure 8 Training load. 


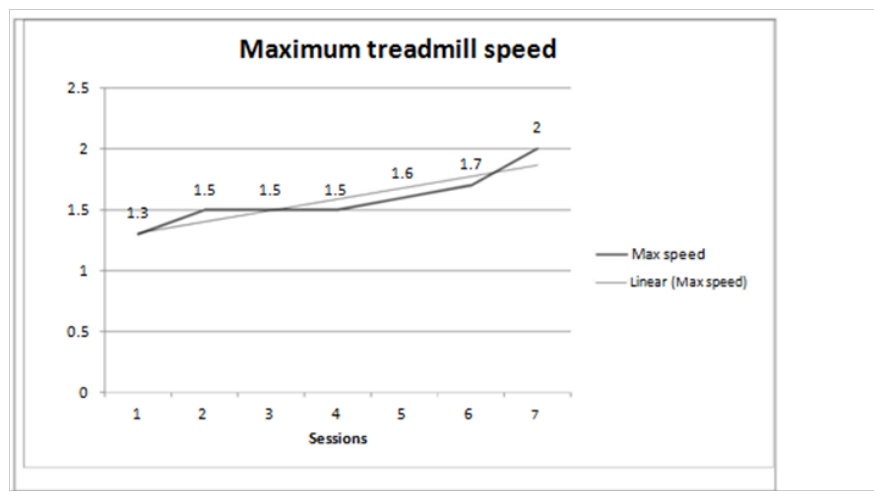

Figure 9 Maximum treadmill speed achieved per session.

\section{Outcomes}

At the end of the BWSTT intervention the patient was reassessed using the same outcome measures as the initial assessment. At reassessment the ABS scale score was $32 \%$ at evaluation and $67 \%$ at discharge demonstrating an improvement of 35 percentage points. Comparison of the initial and final $\mathrm{ABC}$ scale scores and the individual items can be seen in Table 2. The results indicate a significant improvement in her confidence in negotiating many commonly encountered situations without fear of falling.

The TUG test score results can be seen in Table 3. The average of the initial TUG score was 34.36 seconds and 25.66 seconds for the final average, indicating an improvement of 8.7 seconds or $25 \%$, which exceeds the smallest real difference of $23 \%$. Despite the improvement, the TUG scores still indicate the patient is at significant risk for falls. The average 10WMT scores increased from 16.95 seconds to $19.36 \mathrm{M} / \mathrm{S}$ at the final assessment indicate deterioration in gait speed over the course of the intervention. These data are shown in Table 4.

A secondary outcome measure was an assessment of the patient's overall activity and specifically ambulation because of the intervention. The patient was asked to download the Argus application (Azumio, Palo Alto, CA) to measure her steps taken per day, beginning a week before the intervention. The daily step counts as well as a trend line are displayed in Figure 10 and show that there was no real change in her activity during the intervention period.

Table 2 Activities-specific balance confidence scale scores

\begin{tabular}{|c|c|c|c|c|}
\hline \multicolumn{2}{|r|}{ Activities-specific balance confidence (ABC) scale } & \multirow{2}{*}{$\begin{array}{l}\text { Initial } \\
80\end{array}$} & \multirow{2}{*}{$\begin{array}{l}\text { Final } \\
90\end{array}$} & \multirow{2}{*}{$\begin{array}{c}\text { \%Chan } \\
10\end{array}$} \\
\hline I & ...walk around the house? & & & \\
\hline 2 & ...walk up or down stairs? & 60 & 90 & 30 \\
\hline 3 & ...bend over and pick up a slipper from the front of a closet floor? & 50 & 85 & 35 \\
\hline 4 & ...reach for a small can off a shelf at eye level? & 30 & 75 & 45 \\
\hline 5 & ...stand on your tip toes and reach for something above your head? & 30 & 60 & 30 \\
\hline 6 & ...stand on a chair and reach for something? & 90 & 0 & -90 \\
\hline 7 & ...sweep the floor? & 70 & 100 & 30 \\
\hline 8 & ...walk outside the house to a car parked in the driveway? & 100 & 90 & -10 \\
\hline 9 & ...get into or out of a car? & 0 & 100 & 100 \\
\hline 10 & ...walk across a parking lot to the mall? & 0 & 0 & 0 \\
\hline 11 & ...walk up or down a ramp? & 0 & 0 & 0 \\
\hline 12 & ...walk in a crowded mall where people rapidly walk past you? & 0 & 0 & 0 \\
\hline 13 & ...are bumped into by people as you walk through the mall? & 0 & 0 & 0 \\
\hline 14 & ...step onto or off of an escalator while you are holding onto a railing? & 0 & 0 & 0 \\
\hline 15 & $\begin{array}{l}\text {...step onto or off an escalator while holding onto parcels such that you cannot hold onto the } \\
\text { railing? }\end{array}$ & 0 & 0 & 0 \\
\hline \multirow[t]{2}{*}{16} & ...walk outside on icy sidewalks? & 0 & 0 & 0 \\
\hline & SCORE & 32 & 67 & 35 \\
\hline
\end{tabular}


Table 3 Timed Up and Go scores

\begin{tabular}{lllll}
\hline TUG (in seconds) & Initial & Final & Change & SRD \\
\hline Average & 34.36 & 25.66 & 8.7 & $25 \%$
\end{tabular}

Table 4 Ten Meter Walk Test scores

\begin{tabular}{llll}
\hline IOMWT (in seconds) & Initial & Final & Change \\
\hline Average & 16.95 & 19.36 & 2.4 \\
Meters/second & 0.35 & 0.31 & -0.04 \\
Miles per hour & 0.79 & 0.69 & -0.1
\end{tabular}

A follow up gait analysis was performed the final assessment videos. Although technological limitations in the motion analysis software made quantitative comparison difficult, some qualitative comparisons can be made. Through qualitative gait assessment it was noted that the patient continued to display foot flat initial contact on the right (Figure 11) but no longer displayed forward trunk bending or knee hyperextension at midstance and displays trunk rotation with contralateral arm swing (Figure 12). Without the AFO the subject barely clears her toes during midswing (Figure 13) and displays right lower extremity adduction at midstance (Figure 14).

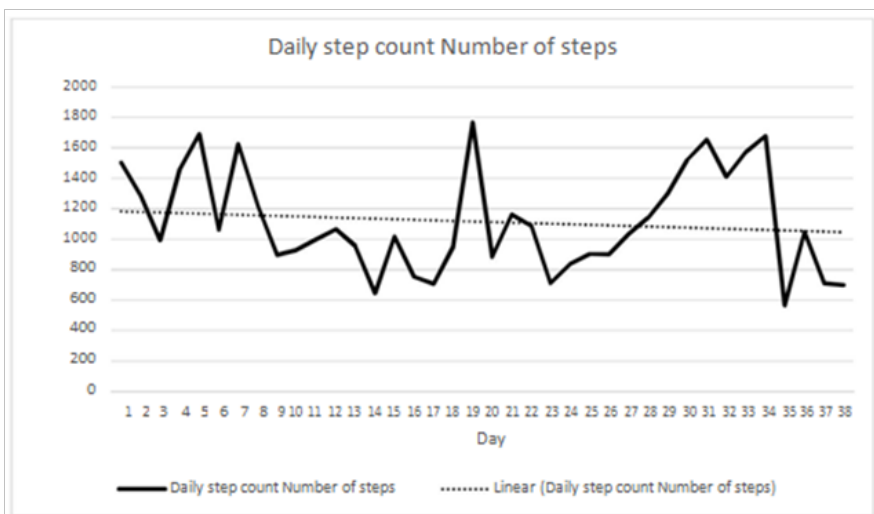

Figure 10 Daily step count.



Figure I I Initial contact.

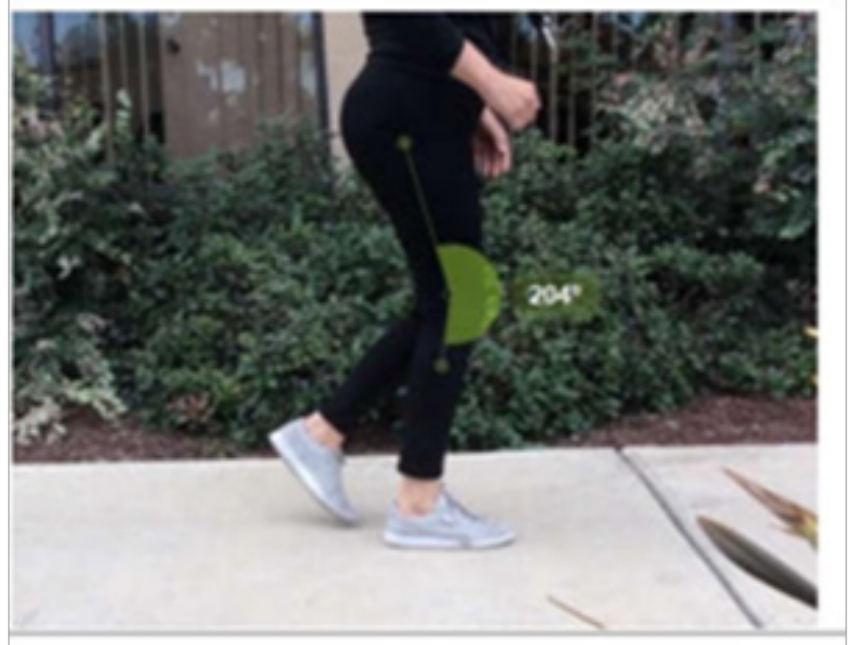

Figure 12 Midstance.

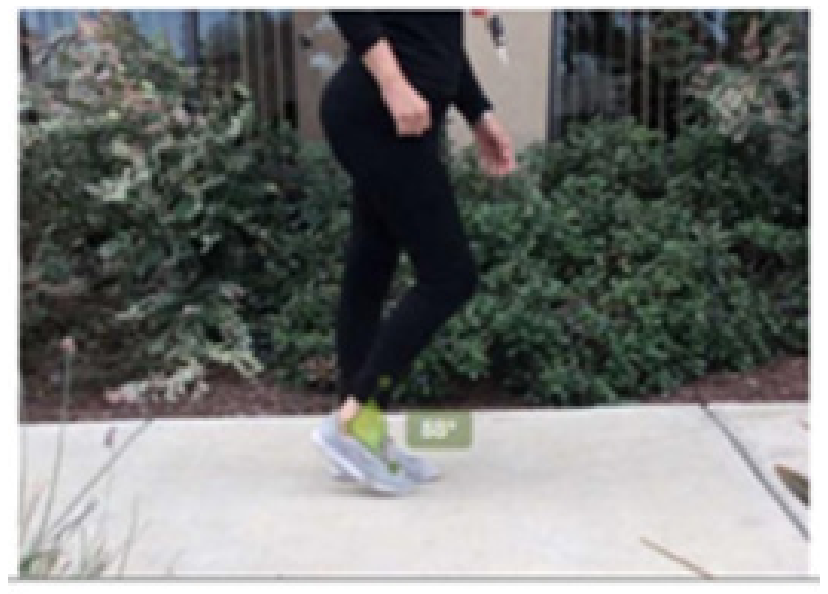

Figure 13 Pre-swing.

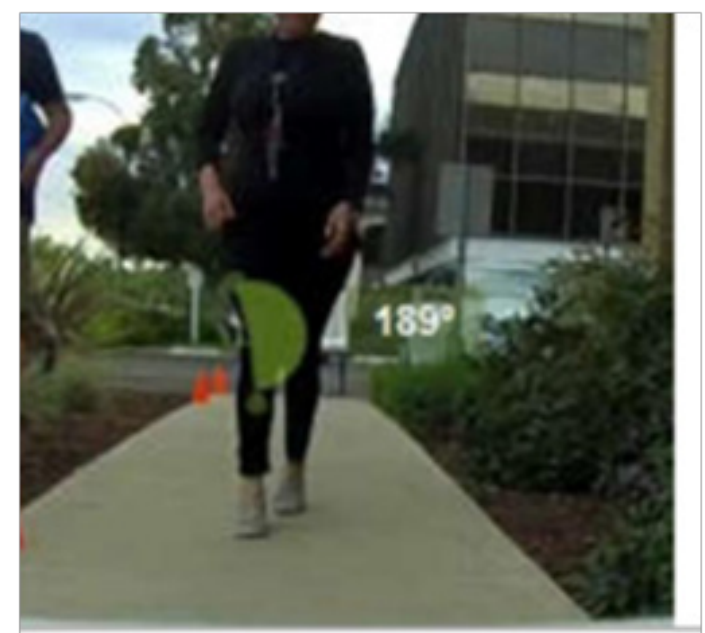

Figure I 4 Midstance. 


\section{Discussion}

Gait speed has been proposed as a "vital sign" for an individual's ability to function as a community ambulator and as a predictor of mortality. ${ }^{22}$ The maximum gait speed as evaluated by the 10 MWT was $0.35 \mathrm{~m} / \mathrm{s}$ at the initial evaluation but $0.31 \mathrm{~m} / \mathrm{s}$ at the final evaluation, demonstrating a $12 \%$ reduction of gait velocity over the course of the intervention. This suggests that BWSTT with the GlideTrak has no carry-over for long term improvement of gait speed.

In contrast, the data from the outcome measures in Tables 2, Table 3 reflect a positive change in the timed up and go and activities-specific balance confidence scale. This data suggest that the body weight treadmill training sessions helped the patient improve in walking around obstacles as well as reducing her fear of falling. Anecdotally, the patient voiced that she felt that her gait and confidence had improved although her gait speed and ability to negotiate obstacles showed no improvement

Lastly, the data collected from the patient's smart phone indicated just how few steps she took during her daily activities during the intervention. The patient averaged between 1000 and 1200 steps per day during the intervention, far below the 5,500 to 6200 steps noted by

Manns and Baldwin in their study of 10 stroke survivors. ${ }^{23}$ One issue in these data is the accuracy of the step count measurement via the smart phone application. The patient reported many spurious readings from the application, once reporting that application showed she had taken over 70 steps when she hadn't yet gotten out of bed. Future studies might be improved with the use of a separate activity monitoring device to get a more accurate picture of activity levels. Additionally, having step counts from before the start of an episode of physical therapy will help the clinician assess the effect of interventions on the patient's quality of life.

There are several limitations in this case report. The setup of the patient on the GlideTrak was conducted by several different personnel, including individuals from the company as well as the author. This may have affected the patient's comfort on the device and her ability to complete the sessions to the limit of fatigue as well as affecting the amount of body weight support and joint angles during ambulation. Secondly, about half of the sessions were performed with the neuroprosthesis AFO and half without because of frequent AFO malfunctioning. Additionally, the AFO malfunctioned frequently during the sessions, having a varied effect on the patient's gait patterns. Consistent use of a properly functioning AFO could have produced different outcomes. Thirdly, the patient received variable amounts of proprioceptive and verbal feedback to correct gait pattern during the sessions. Initially, the patient's right limb was given manual assistance by the GlideTrak personnel and the author to achieve adequate dorsiflexion during swing phase. After a brief period of manual assistance, the patient was able to voluntarily control dorsiflexion and monitor her gait pattern via the sound of her shoes dragging on the treadmill belt. She was occasionally given verbal feedback on her gait pattern, but the feedback appeared to cause the patient to lose concentration. Lastly, there was no way to directly measure the amount of weight bearing during the sessions, which may also have affected the results due to inconsistent amounts of weight bearing during the sessions.
Several external factors could have affected the outcomes of the BWSTT intervention. Although the plan for the case report was to complete 8 sessions, the patient missed one session due to a family emergency and the last session took place after the final assessments were completed, which reduced the training load and perhaps the effect of the intervention. Another possibly confounding factor is that the patient continued her weekly outpatient physical therapy sessions during the intervention. The focus of the treatments was coordinated between the author and the patient's primary physical therapist and concentrated on lower extremity strengthening and gait training to improve her gait pattern. As a result, there were noted improvements in the patient's gait pattern, most notably in arm swing and trunk rotation, toward the end of the BWSTT sessions. Equally important is the possibility of adding two extra sessions of rehabilitation may have caused fatigue, limiting the patient's ability to maximally benefit from the BWSTT.

The GlideTrak system was chosen as an intervention for several reasons. First, the author became familiar with the device while working with one of the original inventors. Secondly, there was no published data on the device's efficacy for improving function in stroke patients. Thirdly, the company's facility is located nearby, and the devices were readily available for the author's use in this study. Lastly, when compared to other BWSTT systems such as the LiteGait or AlterG the GlideTrak system appeared to be easier for patient use as well as requiring less space for home use.

This case report indicates through objective outcome measures that body weight supported treadmill training can benefit stroke patients to improve their mobility and reduce their fear of falling. It may be considered as an important component of rehabilitation along with interventions such as strength, balance and mobility training. As with other BWSTT devices, the GlideTrak requires the facility and/ or patient to have access to the treadmill and the device as well as possibly needing another person to assist with entering and adjusting the support system although the GlideTrak system may be easier than other similar systems. The concomitant use of a neuroprosthetic ankle foot orthosis may offer greater improvements but was not adequately assessed in this report due to limitations noted above.

\section{Acknowledgment}

None.

\section{Conflict of interest}

The Author declares no conflict of interests.

\section{References}

1. Benjamin EJ, Blaha MJ. Heart disease and stroke statistics-2017 update: a report from the american heart association. Circulation. 2017;135(10):e146-e603.

2. Cheung RTF. A systematic approach to the definition of stroke. Austin J Cerebrovasc Dis \& Stroke. 2014;1(5):1024.

3. Kerse N, Parag V, Feigin VL, et al. Falls after stroke results from the auckland regional community stroke (ARCOS) study, 2002 to 2003. Stroke. 2008;39:1890-1893.

4. Studenski S, Perera S, Patel K, et al. Gait speed and survival in older adults. JAMA: the journal of the American Medical Association. 2011;305(1):50-58. 
5. Veerbeek JM, Wegen E, Peppen R, et al. What is the evidence for physical therapy poststroke? A systematic review and meta-analysis. PLoS One. 2014;9(2):e87987.

6. Provost B1, Dieruf K, Burtner PA, et al. Endurance and gait in children with cerebral palsy after intensive body weight-supported treadmill training. Pediatric physical therapy. 2007;19(1):2-10.

7. Mehrholz J, Kugler J, Storch A, et al. Treadmill training for patients with Parkinson's disease. Cochrane Database Syst Rev. 2015;(8):CD007830.

8. Mehrholz J, Kugler J, Pohl M. Locomotor training for walking after spinal cord injury. Cochrane Database Syst Rev. 2012;11:Cd006676.

9. Mehrholz J, Pohl M, Elsner B. Treadmill training and body weight support for walking after stroke. The Cochrane Collaboration, editor. Cochrane Database of Systematic Reviews. 2014;23(1):CD002840.

10. Sullivan KJ, Knowlton BJ, Dobkin BH. Step training with body weight support: effect of treadmill speed and practice paradigms on poststroke locomotor recovery. Arch Phys Med Rehabil. 2002;83:683-691.

11. Charalambous CC, Bonilha HS, Kautz SA, et al. Rehabilitating Walking speed poststroke with treadmill-based interventions: a systematic review of randomized controlled trials. Neurorehabilitation and neural repair. 2013;27(8):709-721.

12. Yen C, Wang R, Liao K, et al. Gait training induced change in corticomotor excitability in patients with chronic stroke. Neurorehabil Neural Repair. 2008;22(1):22-30.

13. Duysens J, Crommert HWAA. Neural control of locomotion; Part 1: The central pattern generator from cats to humans. Gait Posture. 1998;7(2):131-141.
14. Veneri D, Tartaglia J. Determining important dosage parameters to improve gait speed and distance using mechsanical gait support for persons with stroke: a meta-analysis. Critical Reviews in Physical and Rehabilitation Medicine. 2014;26(3-4):255-274.

15. Chen G, Patten C. Treadmill training with harness support: Selection of parameters for individuals with poststroke hemiparesis. The Journal of Rehabilitation Research and Development. 2006;43(4):485-98.

16. https://www.GlideTrak.com

17. Botner EM, Miller WC, Eng JJ. Measurement properties of the activitiesspecific balance confidence scale among individuals with stroke. Disability and Rehabilitation. 2005;27(4):156-163.

18. Hafsteinsdóttir TB, Rensink M, Schuurmans M. Clinimetric properties of the Timed Up and Go Test for patients with stroke: a systematic review. Top Stroke Rehabil. 2014;21(3):197-210.

19. Brach JS, Perera S, Studenski S, et al. Meaningful change in measures of gait variability in older adults. Gait Posture. (2010); 31(2):175-9.

20. Flansbjer UB, Holmbäck AM, Downham D, et al. Reliability of gait performance tests in men and women with hemiparesis after stroke. $J$ Rehabil Med. 2005;37(2):75-82.

21. Althoff T, Sosič R, Hicks J, et al. Large-scale physical activity data reveal worldwide activity inequality. Nature. 2017;547(7663):336-339.

22. Fritz S, Lusardi M. White paper: walking speed: the sixth vital sign. $J$ Geriatr Phys Ther. 2009;32(2):46-9.

23. Manns PJ, Baldwin E. Ambulatory activity of stroke survivors: measurement options for dose, intensity, and variability of activity. Stroke. 2009;40(3):864-7. 